hep-th/0108238

KCL-MTH-01-38

\title{
Conformal boundary states for free bosons and fermions
}

\author{
M.R. Gaberdiel and A. Recknagel \\ Department of Mathematics, King's College London \\ Strand, London WC2R 2LS, U.K.
}

\begin{abstract}
A family of conformal boundary states for a free boson on a circle is constructed. The family contains superpositions of conventional U(1)-preserving Neumann and Dirichlet branes, but for general parameter values the boundary states are fundamental and preserve only the conformal symmetry. The relative overlaps satisfy Cardy's condition, and each boundary state obeys the factorisation constraint. It is also argued that, together with the conventional Neumann and Dirichlet branes, these boundary states already account for all fundamental conformal Dbranes of the free boson theory. The results can be generalised to the situation with $N=1$ world-sheet supersymmetry, for which the family of boundary states interpolates between superpositions of non-BPS branes and combinations of conventional brane anti-brane pairs.
\end{abstract}

August 2001

e-mails: mrg@mth.kcl.ac.uk, anderl@mth.kcl.ac.uk 


\section{Introduction}

Most D-branes that have been constructed in string theory are quite special in that they preserve some large symmetry algebra. For example, the standard Dirichlet or Neumann branes of type IIA or IIB string theory leave all the U(1) current symmetries (associated to the different coordinates in spacetime) invariant. This is to say, the relevant boundary states satisfy the boundary conditions

$$
\left.\left.\left(\alpha_{n}^{\mu} \pm \bar{\alpha}_{-n}^{\mu}\right) \| B\right\rangle\right\rangle=0
$$

where $\alpha_{n}^{\mu}$ are the modes associated to $X^{\mu}$, and where the sign determines whether $X^{\mu}$ obeys a Dirichlet or a Neumann condition on the world-sheet boundary. However, as was already remarked early on in [1], the most general branes that should be relevant for (bosonic) string theory only preserve conformal invariance, i.e. they only satisfy

$$
\left.\left.\left(L_{n}-\bar{L}_{-n}\right) \| B\right\rangle\right\rangle=0 \text {. }
$$

If a boundary state $\| B\rangle\rangle$ satisfies (1.1) for all $\mu$, then (1.2) automatically holds, since the Virasoro modes are bilinears in the current modes. However, in general, (1.2) does not imply (1.1). It is therefore important to understand and characterise the more general class of boundary states that only satisfy (1.2).

In this paper we shall analyse this question in detail for the simplest example, a free boson on a circle. ${ }^{\star}$ We shall also consider the supersymmetric analogue, where we have a free boson and a free fermion. In the bosonic case we shall mainly concentrate on the situation when the radius is a fraction $\frac{M}{N}$ of the self-dual radius (where $M$ and $N$ are coprime), for which we can give an explicit construction of a family of conformal boundary states parametrised by the manifold $\mathrm{SU}(2) / \mathbb{Z}_{M} \times \mathbb{Z}_{N}$. (The action of the cyclic groups is explained in Section 4.) These boundary states have the property that all relative overlaps (boundary partition functions on the strip) satisfy Cardy's condition. We can also provide strong arguments that, together with the standard Dirichlet and Neumann brane states, these boundary states are already all fundamental conformal boundary states. Some of these results had been anticipated by Friedan in unpublished work [3].

The parameter space $\mathrm{SU}(2) / \mathbb{Z}_{M} \times \mathbb{Z}_{N}$ contains two special submanifolds, one describing $M$ Dirichlet branes placed at equidistant circle points $x+\frac{\sqrt{2} \pi k}{N}$ with $k=1, \ldots, M$, while the other submanifold corresponds to superpositions of $N$ Neumann branes with

* For a different class of theories the similar problem of finding boundary states with reduced symmetry was recently analysed in [2]. 
evenly spaced Wilson lines. The general D-branes in the family interpolate between these two extremal configurations. In fact, the Dirichlet or Neumann brane configurations merge into intermediate boundary states that can no longer be thought of as superpositions of fundamental branes. These intermediate branes are themselves fundamental, and they do not preserve the $\mathrm{U}(1)$ symmetry.

In the case with world-sheet supersymmetry, the essential features are the same, although there are a number of minor differences. Most notably, if we want to preserve world-sheet supersymmetry, the boundary states should now also preserve the supercurrent. In addition, in order to obtain a modular invariant partition function for the closed string sector, we have to impose a GSO projection, and this gives rise to additional constraints for the boundary states. The moduli space of superconformal boundary states has essentially the same structure as before, but now the special submanifolds correspond to collections of (unstable) non-BPS branes on the one extreme and to brane anti-brane combinations on the other (the latter are Dirichlet or Neumann depending on the radius $R=M / N)$. As before, the general boundary states in the family interpolate between these configurations. In addition, the moduli space contains an extra branch that describes unstable non-BPS branes similar to those discovered by Sen.

Neither the bosonic nor the fermionic theory are 'rational' with respect to the current or supersymmetric current algebra. The D-branes we consider do not even preserve this algebra, but only the Virasoro or super Virasoro subalgebra; thus they are 'symmetry breaking boundary conditions'. However, in contrast to the framework outlined in [4], our theory is truly non-rational. As a consequence, the usual techniques that are employed for rational theories (in particular, Cardy's construction [5]) are not available in our case. In addition, the question of completeness of the boundary states is not a purely algebraic problem any more, but involves analytic considerations. That one can nevertheless obtain explicit results rests essentially on the fact that irreducible Virasoro representations at $c=1$ are known in great detail.

The paper is organised as follows. In Section 2 we fix our conventions and describe the (conformal) Ishibashi states of the theory. In Section 3 we revisit and reformulate the construction of boundary states for the self-dual radius, and we determine the various overlaps explicitly. These techniques are then generalised in Section 4 to the case where the radius is an arbitrary fraction of the self-dual radius. Section 5 deals with the zero radius limit in which we recover some results of [6], which had been obtained using completely different methods. In Section 6 we analyse the 'factorisation constraints' and show that there are essentially no other fundamental conformal branes. We also explain in some 
detail in which sense these boundary states span the whole space of conformal boundary conditions. Section 7 deals with the modifications that arise in the case with worldsheet supersymmetry, and Section 8 contains some comments regarding the stability of the corresponding D-branes in a ten-dimensional setting. Finally, we end in Section 9 with some open problems.

\section{Notations, conventions and some basic results}

We want to determine a class of conformal boundary states for a free boson of conformal charge $c=1$, and later for the generalisation to the $N=1$ superconformal case. We shall mainly be interested in the situation where the boson is compactified on a circle of radius $R$ (although we shall also make some statements about the limit $R \rightarrow 0$ ). Choosing the convention $\alpha^{\prime}=\frac{1}{2}$, the left- and right-moving momenta are given as

$$
\left(p_{L}, p_{R}\right)=\left(\frac{\hat{m}}{2 R}+\hat{n} R, \frac{\hat{m}}{2 R}-\hat{n} R\right)
$$

with $\hat{m}, \hat{n} \in \mathbb{Z}$. The partition function of the theory is then

$$
Z(q, \bar{q})=\sum_{\hat{m}, \hat{n} \in \mathbb{Z}} \vartheta_{p_{L}(\hat{m}, \hat{n})}(q) \vartheta_{p_{R}(\hat{m}, \hat{n})}(\bar{q})
$$

where we use the slightly non-standard notation

$$
\vartheta_{s}(q)=\frac{q^{\frac{1}{2} s^{2}}}{\eta(q)}
$$

and where $\eta(q)$ is the usual Dedekind $\eta$-function,

$$
\eta(q)=q^{\frac{1}{24}} \prod_{n=1}^{\infty}\left(1-q^{n}\right)
$$

In these units, the self-dual radius is $R_{\mathrm{s.d}}=\frac{1}{\sqrt{2}}$; at this radius, the theory is actually equivalent to a level $k=1$ affine $\widehat{\operatorname{su}}(2)$ theory. (This is the content of the vertex operator construction of Frenkel, Kac and Segal [7,8].)

In the following it will be important to understand the decomposition of the above $\mathrm{U}(1)$ representations in terms of the Virasoro algebra. At $c=1$, every highest weight representation of the Virasoro algebra is irreducible except if the conformal weight is of 
the form $h=j^{2}$ for some $j=0, \frac{1}{2}, 1, \ldots$; in this case, the Virasoro representation has a single null-vector at level $2 j+1[9] .^{\dagger}$ The corresponding characters are then given by

$$
\begin{array}{ll}
h \neq j^{2} & \chi_{h}(q)=\frac{q^{h}}{\eta(q)}, \\
h=j^{2} & \chi_{h}(q)=\vartheta_{\sqrt{2} j}(q)-\vartheta_{\sqrt{2}(j+1)}(q) .
\end{array}
$$

If $p_{L} \neq \sqrt{2} j$ for any $j \in \frac{1}{2} \mathbb{Z}$, then the irreducible U(1)-representation with highest weight $p_{L}$ also defines an irreducible Virasoro representation. On the other hand, if $p_{L}=\sqrt{2} j$ for some $j \in \frac{1}{2} \mathbb{Z}$ we have the decomposition

$$
\mathcal{H}_{j}^{U(1)}=\bigoplus_{l=0}^{\infty} \mathcal{H}_{|j|+l}^{\mathrm{Vir}}
$$

where $\mathcal{H}_{k}^{\text {Vir }}$ (with $k \geq 0$ ) is the irreducible Virasoro representation with highest weight $h=k^{2}$. (This representation can be obtained by dividing out the subrepresentation generated by the null-vector at level $2 k+1$ from the Verma module over the highest weight state.) Here we have used the (obvious) character identity

$$
\vartheta_{\sqrt{2} j}=\sum_{l=0}^{\infty}\left(\vartheta_{\sqrt{2}(j+l)}-\vartheta_{\sqrt{2}(j+l+1)}\right) .
$$

Analogous statements obviously hold for the right-moving representations.

We are only interested in boundary states that preserve the conformal symmetry (so that the corresponding field theory, which is defined on a surface with a boundary, is again conformal). In order to describe the most general such boundary state, we need to rewrite the spectrum of the closed string theory (i.e. the spectrum of the conformal field theory defined on the sphere) in terms of Virasoro representations. For each sector for which the left- and right-moving Virasoro representation are equivalent, we can construct an Ishibashi state; the most general boundary state is then a linear combination of these Ishibashi states, with coefficients constrained by a number of consistency conditions.

In this paper we shall construct a class of boundary states that are built up from Ishibashi states associated with degenerate representations of the Virasoro algebra, i.e. with highest weights $h=j^{2}$ for some $j \in \frac{1}{2} \mathbb{Z}$. The set of representations that are actually

$\dagger$ All of these representations are actually representations of the chiral $c=1$ Virasoro theory (i.e. the chiral vacuum sector or the vertex operator algebra). Since there is a continuum of such representations, the conformal field theory is not 'rational'. Thus, Cardy's technique [5] for finding boundary states does not directly apply. 
present in the closed string spectrum (as well as the multiplicity with which they show up) depends critically on the value of $R$. Degenerate Virasoro representations (other than those in the $\mathrm{U}(1)$ vacuum sector) only occur when $R$ is a fraction of the self-dual radius,

$$
R=\frac{M}{N} \frac{1}{\sqrt{2}},
$$

where $M$ and $N$ are coprime positive integers. In order to describe the set of Ishibashi states for a general radius of the above form, it is convenient to look at the self-dual case $M=N=1$ first (see also $[10,6,11]$ ). In this case, the momenta are of the form

$$
\left(p_{L}, p_{R}\right)=\frac{1}{\sqrt{2}}(\hat{m}+\hat{n}, \hat{m}-\hat{n}),
$$

and the corresponding Virasoro highest weights are given by

$$
h=\frac{1}{2} p_{L}^{2}=\left(\frac{\hat{m}+\hat{n}}{2}\right)^{2}=m^{2}, \quad \bar{h}=\frac{1}{2} p_{R}^{2}=\left(\frac{\hat{m}-\hat{n}}{2}\right)^{2}=n^{2},
$$

where we have defined

$$
m=\frac{\hat{m}+\hat{n}}{2} \quad n=\frac{\hat{m}-\hat{n}}{2} .
$$

By construction, $m$ and $n$ are half-integers whose difference is always integer. Because of (2.10), all the $\mathrm{U}(1)$ representations that occur at the self-dual point give rise to degenerate Virasoro representations. It then follows from (2.6) that for fixed $(m, n)$, the $\mathrm{U}(1) \times \mathrm{U}(1)$ representation (whose highest weight states have momenta $\left(p_{L}, p_{R}\right)$ ) contains the Virasoro representation $\mathcal{H}_{j}^{\text {Vir }} \otimes \overline{\mathcal{H}}_{j}^{\text {Vir }}$ provided that both $|m|$ and $|n|$ are less or equal than $j$ and that $j-m$ (and $j-n)$ is integer. Conversely, if $(m, n)$ satisfy these constraints, the representation $\mathcal{H}_{j}^{\text {Vir }} \otimes \overline{\mathcal{H}}_{j}^{\text {Vir }}$ appears precisely once in the corresponding $\mathrm{U}(1) \times \mathrm{U}(1)$ representation. Thus the possible Ishibashi states can be labelled by the triples $(j ; m, n)$ where $j$ is a non-negative half-integer, $m=-j,-j+1, \ldots, j$, and similarly for $n$. These triples can be thought of as labelling the possible matrix elements of the $\mathrm{SU}(2)$ representation $j$.

For the more general case (2.8), left- and right-moving momenta are of the form

$$
\left(p_{L}, p_{R}\right)=\frac{1}{\sqrt{2}}\left(\frac{\hat{n} N}{M}+\frac{\hat{m} M}{N}, \frac{\hat{n} N}{M}-\frac{\hat{m} M}{N}\right)=\sqrt{2}(m, n),
$$

where $m$ and $n$ are defined in analogy to (2.11), and thus the conformal weights are

$$
h=\frac{1}{4}\left(\frac{\hat{n} N}{M}+\frac{\hat{m} M}{N}\right)^{2}=m^{2} \quad \bar{h}=\frac{1}{4}\left(\frac{\hat{n} N}{M}-\frac{\hat{m} M}{N}\right)^{2}=n^{2} .
$$

In general, $m$ and $n$ are not half-integers any more, and therefore also Ishibashi states for non-degenerate Virasoro representations occur. If we concentrate on the degenerate 
Virasoro representations, i.e. if we choose $\hat{m}, \hat{n}$ so that $m$ and $n$ are half-integers, then $m-n$ and $m+n$ are necessarily integers. (This follows from the fact that $(m-n)(m+n)=\hat{m} \hat{n}$.) Furthermore, since $M$ and $N$ are coprime, we have the constraints

$$
m+n=l N, \quad m-n=l^{\prime} M \quad \text { for some } l, l^{\prime} \in \mathbb{Z}
$$

on the $(m, n)$ that correspond to degenerate Virasoro representations. Conversely, if these constraints are satisfied, the spectrum contains a Virasoro Ishibashi state corresponding to $j$ for every $j$ that is greater or equal to $|m|$ and $|n|$ and satisfies $j-m \in \mathbb{Z}$. Thus the (degenerate) Ishibashi states that exist in the general case can be labelled by the triples $(j ; m, n)$ as before where now $m$ and $n$ satisfy in addition $(2.14)$; this characterisation of the Ishibashi states will be crucial in what follows.

\section{The boundary states at the self-dual radius}

As a warm-up, let us first analyse the boundary states for the theory at the self-dual radius. Explicit expressions were for example given in $[12,11]$, where it was observed that there exists a moduli space of boundary conditions that are connected by truly marginal boundary fields and that can be parametrised by $g \in \mathrm{SU}(2)$. All the boundary states $\| g\rangle\rangle$ in this $S^{3}$-family preserve $\widehat{\mathrm{su}}(2)_{1}$, with gluing conditions

$$
\left.\left.\left.\operatorname{Ad}_{(g \cdot \iota)}\left(J_{m}^{a}\right)+J_{-m}^{a}\right) \| g\right\rangle\right\rangle=0 \quad \text { where } \quad \iota=\left(\begin{array}{cc}
0 & 1 \\
-1 & 0
\end{array}\right)
$$

and for all $m \in \mathbb{Z}$. In the following we shall concentrate on checking the Cardy condition (which was only implicitly solved in [11]). In particular, we shall obtain a compact formula for the open string spectrum between arbitrary boundary states. A modification of the technique we shall use in the derivation of this result can then be used to solve the Cardy condition for all radii of the form (2.8).

For each group element $g \in \mathrm{SU}(2)$, we define the boundary state

$$
\left.\| g\rangle\rangle=\frac{1}{2^{\frac{1}{4}}} \sum_{j, m, n} D_{m, n}^{j}(g)|j ; m, n\rangle\right\rangle,
$$

where $D_{m, n}^{j}(g)$ is the matrix element of $g$ in the representation $j$, and $\left.|j ; m, n\rangle\right\rangle$ denotes the Virasoro Ishibashi state labelled by the triple $(j ; m, n)$ as above. The gluing condition

fixes the Ishibashi state up to an overall factor; we shall always assume that they are normalised so that

$$
\left\langle\left\langle j ; m, n\left|q^{\frac{1}{2}\left(L_{0}+\bar{L}_{0}-\frac{c}{12}\right)}\right| j ; m, n\right\rangle\right\rangle=\chi_{j^{2}}(q),
$$


where $\chi_{j^{2}}$ denotes the character of the Virasoro highest weight representation with $h=j^{2}$ (see (2.5) above). An explicit formula for the matrix elements is given by

$$
\begin{aligned}
D_{m, n}^{j}(g)= & \sum_{l=\max (0, n-m)}^{\min (j-m, j+n)} \frac{[(j+m) !(j-m) !(j+n) !(j-n) !]^{\frac{1}{2}}}{(j-m-l) !(j+n-l) ! l !(m-n+l) !} \\
& \times a^{j+n-l}\left(a^{*}\right)^{j-m-l} b^{m-n+l}\left(-b^{*}\right)^{l}
\end{aligned}
$$

where we have written $g \in \mathrm{SU}(2)$ as

$$
g=\left(\begin{array}{cc}
a & b \\
-b^{*} & a^{*}
\end{array}\right)
$$

This differs slightly from the formula given in [13]; with the definition (3.4) we then have $D_{m, n}^{j}(g \cdot h)=\sum_{l} D_{m, l}^{j}(g) D_{l, n}^{j}(h)$.

If we set $g=e$ in (3.2), the boundary state describes the standard Dirichlet brane (at $x=0$ ). On the other hand, the standard Neumann brane (without any Wilson line) corresponds to the choice $a=0, b=1$ in (3.5). The prefactor of (3.2) is the familiar factor $(2 R)^{-\frac{1}{2}}$ that arises in the normalisation of conventional Dirichlet boundary states.

One of the consistency conditions that have to be satisfied by the boundary states is the constraint - usually referred to as the Cardy condition [5] - that the overlap of any two boundary states gives rise, upon modular transformation, to an open string oneloop amplitude. In particular, this requires that the different 'open string representations' contributing to the one-loop amplitude occur with positive integer multiplicities. We shall now demonstrate explicitly that the boundary states (3.2) satisfy this constraint. In order to do so, we begin by rewriting the closed string tree-level amplitude

$$
\mathcal{A}=\left\langle\left\langle g_{1}\left\|q^{\frac{1}{2}\left(L_{0}+\bar{L}_{0}-\frac{c}{12}\right)}\right\| g_{2}\right\rangle\right\rangle=\frac{1}{\sqrt{2}} \sum_{j \in \frac{1}{2} \mathbb{Z}_{+}} \sum_{m, n}\left(D_{m, n}^{j}\left(g_{1}\right)\right)^{*} D_{m, n}^{j}\left(g_{2}\right) \chi_{j^{2}}(q) .
$$

Since the representation defined by $j$ is unitary, we have

$$
\left(D_{m, n}^{j}\left(g_{1}\right)\right)^{*}=D_{n, m}^{j}\left(g_{1}^{-1}\right)
$$

For each fixed $j$, we can then perform the sum over $m$ and $n$, and we obtain

$$
\sum_{m, n} D_{n, m}^{j}\left(g_{1}^{-1}\right) D_{m, n}^{j}\left(g_{2}\right)=\sum_{n} D_{n, n}^{j}\left(g_{1}^{-1} g_{2}\right)=\operatorname{Tr}_{j}\left(g_{1}^{-1} g_{2}\right)
$$

where $\operatorname{Tr}_{j}$ is the trace in the $j$-th representation of $\mathrm{SU}(2)$. Since the trace is invariant under conjugation, we can rotate $g_{1}^{-1} g_{2}$ to lie in the maximal torus of $\mathrm{SU}(2)$; then

$$
\operatorname{Tr}_{j}\left(g_{1}^{-1} g_{2}\right)=\frac{\sin ((2 j+1) \alpha)}{\sin \alpha}
$$


for some $\alpha \in[0,2 \pi)$ that depends on $g_{1}^{-1} g_{2}$. (The simplest way to determine $\alpha$ explicitly is to evaluate the left hand side of (3.9) in the fundamental $\left(j=\frac{1}{2}\right)$ representation of $\mathrm{SU}(2)$, where the right hand side is simply $2 \cos \alpha$.)

Next, we rewrite the Virasoro character $\chi_{j^{2}}$ in terms of the $\vartheta$-functions as in (2.5), and thus obtain

$$
\begin{aligned}
\sqrt{2} \mathcal{A}= & \sum_{j \in \frac{1}{2} \mathbb{Z}_{+}} \frac{\sin ((2 j+1) \alpha)}{\sin \alpha}\left(\vartheta_{\sqrt{2} j}(q)-\vartheta_{\sqrt{2}(j+1)}(q)\right) \\
= & \vartheta_{0}(q)+\sum_{j=1}^{\infty} \vartheta_{\sqrt{2} j}(q)\left(\frac{\sin ((2 j+1) \alpha)}{\sin \alpha}-\frac{\sin ((2 j-1) \alpha)}{\sin \alpha}\right) \\
& +2 \cos \alpha \cdot \vartheta_{\frac{1}{\sqrt{2}}}(q)+\sum_{j=\frac{3}{2}}^{\infty} \vartheta_{\sqrt{2} j}(q)\left(\frac{\sin ((2 j+1) \alpha)}{\sin \alpha}-\frac{\sin ((2 j-1) \alpha)}{\sin \alpha}\right) \\
= & \sum_{j \in \frac{1}{2} \mathbb{Z}} \cos (2 j \alpha) \vartheta_{\sqrt{2} j}(q),
\end{aligned}
$$

where the first (second) sum in the middle equation runs over integers (half-odd integers) only. Moreover, we have used the identity

$$
\frac{\sin ((2 j+1) \alpha)}{\sin \alpha}-\frac{\sin ((2 j-1) \alpha)}{\sin \alpha}=2 \cos (2 j \alpha),
$$

as well as the fact that $\vartheta_{s}(q)$ only depends on $|s|$. Under a modular transformation, the $\vartheta$-functions (2.3) behave as

$$
\vartheta_{s}(q)=\int_{-\infty}^{\infty} d t e^{2 \pi i t s} \vartheta_{t}(\tilde{q})
$$

where $q=e^{2 \pi i \tau}$ with $\Im \tau>0$ and $\tilde{q}=e^{-\frac{2 \pi i}{\tau}}$. Putting these results together, we then find that the overlap $\mathcal{A}=Z_{g_{1} g_{2}}(\tilde{q})$ becomes, in the open string description,

$$
\begin{aligned}
Z_{g_{1} g_{2}}(\tilde{q}) & =\frac{1}{\sqrt{2}} \int_{-\infty}^{\infty} d t \vartheta_{t}(\tilde{q}) \sum_{j \in \frac{1}{2} \mathbb{Z}} e^{2 \pi i \sqrt{2} j t} \cos (2 j \alpha) \\
& =\frac{1}{\sqrt{2}} \int_{-\infty}^{\infty} d t \vartheta_{t}(\tilde{q}) \sum_{l \in \mathbb{Z}} e^{i l(\sqrt{2} \pi t+\alpha)} \\
& =\frac{2 \pi}{\sqrt{2}} \int_{-\infty}^{\infty} d t \vartheta_{t}(\tilde{q}) \sum_{n \in \mathbb{Z}} \delta(\sqrt{2} \pi t+\alpha+2 \pi n) \\
& =\sum_{n \in \mathbb{Z}} \vartheta_{-\frac{\alpha}{\sqrt{2} \pi}+\sqrt{2} n}(\tilde{q}) .
\end{aligned}
$$


Irrespective of the value of $\alpha$, this defines a positive integer linear combination of Virasoro or $\mathrm{U}(1)$ characters. For $g_{1}=g_{2}$, the spectrum displays the full $\widehat{\operatorname{su}}(2)_{1}$-symmetry as expected.

Since $\alpha$ only depends on $g_{1}^{-1} g_{2}$, the open string spectrum between any of the above Dirichlet branes (labelled by $g \in \mathrm{SU}(2)$ ) and itself is the same for all $g$. This agrees with the results found in $[10,6,11]$. However, (3.13) also determines the open string spectrum between two D-branes associated to different SU(2) elements.

\section{The generalisation to fractional radii}

We shall now show that, for any 'fractional radius' of the form $R=\frac{M}{N} R_{\text {s.d. }}$ as in (2.8), there is again a conformal boundary state for each group element $g \in \mathrm{SU}(2)$. These boundary states are given by formula (3.2) from above, except for the following two changes: (i) the $m$ - and $n$-summations run only over values which satisfy in addition (2.14), and (ii) the overall normalisation is $\sqrt{M N}$ times larger than the one in (3.2). Since for $R \neq R_{\text {s.d., }}$, the Virasoro Ishibashi states do not align into U(1) Ishibashi states, the new boundary states will in general break the symmetry from $\mathrm{U}(1)$ to Vir. If $M=1$, these states can be obtained by marginal boundary deformations of an ordinary Dirichlet boundary state $[10,6,11]$, and the analogous statement holds for the $T$-dual situation. For other radii, they are not connected to a single U(1)-preserving Dirichlet boundary state by marginal deformations; however, as we shall see below, they are connected to certain superpositions of ordinary Dirichlet branes.

In order to show that the corresponding open string spectrum still satisfies the desired integrality property, we want to perform a calculation similar to what we have done in the previous section. However, there is one new difficulty, namely that (3.8) is not applicable as it stands since we are not summing over all values of $m$ and $n$ any more. We can circumvent this problem if we use the following trick. Let us introduce the group element

$$
\Gamma_{M}=\left(\begin{array}{cc}
e^{\frac{\pi i}{M}} & 0 \\
0 & e^{-\frac{\pi i}{M}}
\end{array}\right) \in \mathrm{SU}(2) .
$$

Using the explicit form of (3.4) we find that

$$
D_{m, n}^{j}\left(\Gamma_{M}\right)=e^{\frac{2 \pi i}{M} m} \delta_{m, n}
$$

Thus,

$$
D_{m, n}^{j}\left(\Gamma_{M}^{l} g \Gamma_{M}^{-l}\right)=e^{\frac{2 \pi i}{M} l(m-n)} D_{m, n}^{j}(g),
$$


and therefore

$$
P_{M}^{-}(g):=\frac{1}{M} \sum_{l=0}^{M-1} D_{m, n}^{j}\left(\Gamma_{M}^{l} g \Gamma_{M}^{-l}\right)=\left\{\begin{aligned}
D_{m, n}^{j}(g) & \text { if } m-n \equiv 0(\bmod M) \\
0 & \text { otherwise. }
\end{aligned}\right.
$$

Similarly, we have

$$
P_{N}^{+}(g):=\frac{1}{N} \sum_{k=0}^{N-1} D_{m, n}^{j}\left(\Gamma_{N}^{k} g \Gamma_{N}^{k}\right)=\left\{\begin{aligned}
D_{m, n}^{j}(g) & \text { if } m+n \equiv 0(\bmod N) \\
0 & \text { otherwise. }
\end{aligned}\right.
$$

Hence, if we replace $D_{m, n}^{j}(g)$ by

$$
P_{N}^{+} P_{M}^{-}(g)=\frac{1}{M N} \sum_{l=0}^{M-1} \sum_{k=0}^{N-1} D_{m, n}^{j}\left(\Gamma_{N}^{k} \Gamma_{M}^{l} g \Gamma_{M}^{-l} \Gamma_{N}^{k}\right)
$$

we can actually drop the restriction (2.14) on $m$ and $n$, and we arrive at the following expression for our symmetry-breaking boundary states

$$
\left.\| g\rangle\rangle_{M, N}=2^{-\frac{1}{4}} \sqrt{M N} \sum_{j, m, n} D_{m, n}^{j}\left(P_{N}^{+} P_{M}^{-}(g)\right)|j ; m, n\rangle\right\rangle
$$

We have made the radius $R=\frac{M}{N} R_{\text {s.d. }}$ explicit for the moment, and have inserted the normalisation factor $\sqrt{M N}$ mentioned above. Upon computing the overlap between two such boundary states, each term in the $(k, l)$-sum from $(4.6)$ will give rise to an open string spectrum of the form (3.13), and we obtain

$$
Z_{g_{1} g_{2}}(\tilde{q})=\sum_{l=0}^{M-1} \sum_{k=0}^{N-1} \sum_{n \in \mathbb{Z}} \vartheta_{-\frac{\alpha_{k l}\left(g_{1}, g_{2}\right)}{\sqrt{2} \pi}+\sqrt{2} n}(\tilde{q})
$$

where

$$
2 \cos \left(\alpha_{k l}\left(g_{1}, g_{2}\right)\right)=\operatorname{Tr}_{\frac{1}{2}}\left[g_{1}^{-1} \Gamma_{N}^{k} \Gamma_{M}^{l} g_{2} \Gamma_{M}^{-l} \Gamma_{N}^{k}\right]
$$

the trace being taken in the fundamental representation $j=\frac{1}{2}$. Equation (4.9) only determines $\alpha_{k, l}$ up to a multiple of $2 \pi$. However, the partition function $Z_{g_{1} g_{2}}$ is independent of this ambiguity because of the summation over $n$. The partition function (4.8) defines a positive integer linear combination of Virasoro characters, and thus Cardy's conditions are met.

The spectrum seems to exhibit 'traces' of the underlying $U(1)$ bulk symmetry in a similar way as the spectrum of open strings stretched between two non-coinciding Dirichlet branes. But in contrast to the latter situation, complete $\mathrm{U}(1)$ symmetry is not even restored 
in the excitation spectrum of a single brane $\| g\rangle\rangle_{M, N}$, for generic $M, N$ : if we consider the case for which $g_{1}=g_{2}=g$ with $g$ as in (3.5), then (4.9) simplifies to

$$
\cos \left(\alpha_{k l}(g)\right)=\cos \left(\frac{2 \pi k}{N}\right) a^{*} a+\cos \left(\frac{2 \pi l}{M}\right) b^{*} b .
$$

Clearly, the spectrum encoded in $Z_{g g}(\tilde{q})$ is not, in general, invariant under addition of 'U(1)-charges'. This shows once more that the boundary states $\| g\rangle\rangle_{M, N}$ do indeed break the $\mathrm{U}(1)$ symmetry.

In order to make contact with results obtained previously in the literature, let us specialise to $\mathrm{SU}(2)$ elements of the form

$$
g=g_{\lambda}:=\left(\begin{array}{cc}
\cos \lambda & \sin \lambda \\
-\sin \lambda & \cos \lambda
\end{array}\right)
$$

and to the case $N=2, M=1$. Then the angles from above read

$$
\alpha_{00}(g)=0, \quad \alpha_{10}(g)=2 \lambda+\pi
$$

and inserting these back into (4.8) reproduces the spectrum found in [11] in connection with truly marginal, symmetry-breaking deformations of a Dirichlet boundary state.

Let us study the case $g_{1}=g_{2}=g$ further in order to establish an interpretation of our family of boundary states $\| g\rangle\rangle_{M, N}$. First, one notices that the spectrum $Z_{g g}(\tilde{q})$ contains a single vacuum state unless the elements of the $\mathrm{SU}(2)$ matrix $g$ satisfy $b=0$ or $a=0$. Along those two curves in the 3-parameter space, the vacuum occurs with $M$-fold (or $N$-fold) degeneracy. This effect is easy to understand since, for these values of $g$, our boundary states can be expressed as superpositions of ordinary U(1) Dirichlet (or Neumann) boundary states, $\left.\left.\| D, x_{0}\right\rangle\right\rangle$ (or $\left.\left.\| N, \tilde{x}_{0}\right\rangle\right\rangle$ ), at special (equidistant) values of locations $x_{0}$ (or Wilson lines $\tilde{x}_{0}$ ); in our conventions,

$$
\begin{aligned}
\left.\left.\|\left(\begin{array}{cc}
1 & 0 \\
0 & 1
\end{array}\right)\right)\right\rangle_{M, N} & \left.\left.=\sum_{p=0}^{M-1} \| D, \frac{2 \pi R p}{M}\right\rangle\right\rangle, \\
\left.\left.\|\left(\begin{array}{cc}
0 & 1 \\
-1 & 0
\end{array}\right)\right)\right\rangle_{M, N} & \left.\left.=\sum_{s=0}^{N-1} \| N, \frac{\pi s}{R N}\right\rangle\right\rangle .
\end{aligned}
$$

As an aside, it follows by essentially the same observation that the overlap between the boundary states labelled by $g \in \mathrm{SU}(2)$ and the conventional U(1) Dirichlet and Neumann boundary states also give rise to an integer linear combination of Virasoro characters in the open string (and therefore that they also satisfy the mutual Cardy conditions). 
On the other hand, there is no simple relation between the $\mathrm{U}(1)$ boundary states and our boundary states for generic $g$ (i.e. for $a b \neq 0$ ). In this case, $Z_{g g}(\tilde{q})$ always contains three states of conformal dimension $h=1$ : one from the $\mathrm{U}(1)$ vacuum character $\vartheta_{0}(\tilde{q})$ and one each from the two characters $\vartheta_{ \pm \sqrt{2}}(\tilde{q})$. For the cases $R=M R_{\text {s.d. }}$ and $R=\frac{1}{N} R_{\text {s.d. }}$, the corresponding marginal boundary fields were studied in [11] and argued to be truly marginal operators. In fact, one of these operators is associated to motions of the brane (or changes of the Wilson line), while the other two correspond to turning on boundary potentials as in $[10,6]$. The situation at hand is technically slightly more complicated since one has to deal with boundary condition changing operators in order to study deformations of superpositions of Dirichlet or Neumann branes. Nevertheless, the main arguments used in [11] for the existence of a 3-parameter family of truly marginal operators should carry over to the general case $N \neq 1, M \neq 1$. We therefore conjecture that the marginal operators present here enjoy analogous properties, and that our family of boundary states is indeed connected by truly marginal deformations. Since, for generic $g \in \mathrm{SU}(2)$, the boundary conditions $\| g\rangle\rangle$ are 'elementary' in the sense that the vacuum occurs precisely once in $Z_{g g}(\tilde{q})$, one is led to the conclusion that these deformations merge a set of $M$ equidistant Dirichlet branes into a single brane (of neither Dirichlet nor Neumann type, and preserving only the Virasoro algebra), until upon further deformation one reaches a system of $N$ Neumann branes with evenly spaced Wilson lines.

One should, however, note that our parametrisation of boundary states by $\mathrm{SU}(2)$ elements chosen above is not quite one-to-one if $M \neq 1$ or $N \neq 1$. From the definition of the representation matrices (3.4) it follows that

$$
D_{m, n}^{j}(g)=e^{-i \theta(m+n)} D_{m, n}^{j}\left(g\left(a_{\theta}\right)\right)=e^{-i \varphi(m-n)} D_{m, n}^{j}\left(g\left(b_{\varphi}\right)\right),
$$

where $g\left(a_{\theta}\right)$ is the matrix that is obtained from $g$ by replacing $a$ by $e^{i \theta} a$ in (3.5), while $g\left(b_{\varphi}\right)$ is the matrix that is obtained by replacing $b$ by $e^{i \varphi} b$. For the boundary states we have constructed, only those matrix elements arise for which $m+n$ is a multiple of $N$, and $m-n$ is a multiple of $M$. Thus the brane that corresponds to $g$ is the same as the ones described by $g\left(a_{\theta}=2 \pi \frac{l}{N}\right)$ or $g\left(b_{\varphi}=2 \pi \frac{l^{\prime}}{M}\right)$ for any integers $l, l^{\prime}$. For the cases $M=1$ or $N=1$, the discrete identifications described above were overlooked in [11]. These are the only non-trivial identifications since (3.4) also implies that

$$
D_{j, j}^{j}(g)=a^{2 j}, \quad D_{j,-j}^{j}(g)=b^{2 j},
$$

so all other changes of $a$ and $b$ would be detected by the matrix elements that occur in the boundary states. Thus we conclude that the moduli space of our new boundary states at $R=\frac{M}{N} R_{\text {s.d. }}$ is given by the quotient

$$
\mathcal{M}_{M, N}=\mathrm{SU}(2) / \mathbb{Z}_{M} \times \mathbb{Z}_{N}
$$




\section{The zero radius limit}

We can use the trick described in the previous subsection to (re)derive the formula for the open string spectrum (between a brane and itself, say) in the zero radius limit (or similarly for $R \rightarrow \infty)$. The zero radius limit corresponds to talking $N \rightarrow \infty$ while $M=1$. The relevant projector that replaces (4.6) is then given by the integral

$$
\frac{1}{\pi} \int_{0}^{\pi} d \theta D_{m, n}^{j}\left(\left(\begin{array}{cc}
e^{i \theta} & 0 \\
0 & e^{-i \theta}
\end{array}\right) g\left(\begin{array}{cc}
e^{i \theta} & 0 \\
0 & e^{-i \theta}
\end{array}\right)\right) .
$$

(In order to deal with $R \rightarrow \infty$, one simply replaces $\theta$ by $-\theta$ in the last matrix.) Up to an overall normalisation (which is formally infinite) the relevant tree amplitude is then

$$
\mathcal{A}=\frac{1}{\pi} \int_{0}^{\pi} d \theta \sum_{j \in \frac{1}{2} \mathbb{Z}_{+}} \operatorname{Tr}_{j}\left[\left(\begin{array}{cc}
e^{i \theta} & 0 \\
0 & e^{-i \theta}
\end{array}\right) g\left(\begin{array}{cc}
e^{i \theta} & 0 \\
0 & e^{-i \theta}
\end{array}\right) g^{-1}\right] \chi_{j^{2}}(q) .
$$

For fixed $g$ as in (3.5), and for each value of $\theta$, the trace in (5.2) can be written as $\frac{\sin ((2 j+1) \alpha)}{\sin \alpha}$ for some $\alpha \equiv \alpha(g, \theta)$. In order to determine $\alpha$, we work in the fundamental representation $\left(j=\frac{1}{2}\right)$, for which we obtain

$$
\begin{aligned}
2 \cos \alpha & =\operatorname{tr}\left[\left(\begin{array}{cc}
e^{2 i \theta} a & b \\
-b^{*} & e^{-2 i \theta} a^{*}
\end{array}\right)\left(\begin{array}{cc}
a^{*} & -b \\
b^{*} & a
\end{array}\right)\right] \\
& =2\left[1+a^{*} a(\cos (2 \theta)-1)\right] .
\end{aligned}
$$

Using trigonometric identities and taking square roots on both sides, (5.3) becomes

$$
\sin \left(\frac{\alpha}{2}\right)=|a| \sin \theta
$$

and thus the angles in the partition function can be written as $\alpha=2 \pi f(g, \theta)$ with

$$
f(g, \theta)=\frac{1}{\pi} \arcsin (|a| \sin \theta) .
$$

Inserting this into our previous calculation, the open string spectrum is then given by

$$
Z_{g, g}=\frac{1}{\eta(\tilde{q})} \sum_{n \in \mathbb{Z}} \int_{0}^{\pi} \frac{d \theta}{\pi} \tilde{q}^{(n+f(g, \theta))^{2}} .
$$

In the special case $g=g_{\lambda}$ from (4.11), this agrees with the result obtained in [6] using fermionisation techniques. 


\section{The factorisation property, completeness, and remarks on non-rational radii}

All the boundary states we have considered so far have the property that, up to some irrelevant overall constant, the coefficients in front of the various Ishibashi states are given in terms of the matrix elements $D_{m, n}^{j}(g)$ and therefore satisfy

$$
D_{m_{1}, n_{1}}^{j_{1}}(g) D_{m_{2}, n_{2}}^{j_{2}}(g)=\sum_{j=\left|j_{1}-j_{2}\right|}^{j_{1}+j_{1}} C_{j_{1}, j_{1}}^{j}\left(m_{1}, n_{1} ; m_{2}, n_{2}\right) D_{m_{1}+m_{2}, n_{1}+n_{2}}^{j}(g),
$$

where $C_{j_{1}, j_{1}}^{j}\left(m_{1}, n_{1} ; m_{2}, n_{2}\right)$ are products of Clebsch-Gordan coefficients (see [14] for more details). Indeed, the left hand side of (6.1) is the matrix element between the states labelled by $\left(m_{1} \otimes m_{2}\right)$ and $\left(n_{1} \otimes n_{2}\right)$ in the tensor product of the representations $j_{1}$ and $j_{2}$; the Clebsch-Gordan coefficients describe the decomposition of this tensor product into irreducible representations, which yields (6.1).

As follows from the detailed explanation in [14], the boundary structure constants $B_{(j ; m, n)}^{g} \equiv{ }^{g} B_{(j ; m, n)}^{\mathbb{1}}$ from the bulk-boundary OPE in the presence of the boundary condition $\| g\rangle\rangle$ are given by $D_{m, n}^{j}\left(\iota \cdot g \cdot \iota^{-1}\right)$, where $\iota=\left(\begin{array}{cc}0 & 1 \\ -1 & 0\end{array}\right)$. Therefore, eq. (6.1) has the same form as the sewing constraint that arises from equating two different ways of evaluating a two-point function of bulk fields on the upper half-plane: in one factorisation, the two bulk fields become arbitrary close to each other and the leading term is determined by the bulk operator product expansion, while in the other the two bulk fields come separately arbitrarily close to the boundary and the leading term is obtained from bulk-boundary operator product expansions. The general form of this constraint, which can also be understood as a cluster condition, is

$$
B_{a}^{\alpha} B_{b}^{\alpha}=\sum_{c} \Xi_{a b c} B_{c}^{\alpha}
$$

where (up to an $a$-independent factor) $B_{a}^{\alpha}$ is the coefficient of the Ishibashi state associated to the representation $a$ in the boundary state labelled by $\alpha$ (in our case, $a=(j ;, m, n)$ ), while $\Xi_{a b c}$ is a product of the bulk OPE coefficient $C_{a b c}$ with an element of the fusing matrix. In writing $(6.2)$, the normalisation $B_{0}^{\alpha}=1$ for the vacuum sector has been chosen; see e.g. $[15,16,17,18,11]$ for more details.

Comparison of this constraint with (6.1) suggests that in our case the $\Xi$-sometimes also referred to as the structure constants of the 'classifying algebra' [17] - are precisely given by products of Clebsch-Gordan coefficients. As is explained in detail in [14], at the self-dual radius the structure constants are indeed given by these group theoretic expressions. We are only interested in the Virasoro symmetry here, and the different representations are therefore completely characterised by their conformal weight. Since 
these are independent of the radius $R$, we can conclude that these structure constants do not depend on $R$, and therefore that the boundary states we have constructed satisfy all factorisation constraints that involve degenerate Virasoro representations only. It seems plausible to us that the boundary states also satisfy the other factorisation constraints (those involving two non-degenerate representations and one degenerate representation) provided that the group element $g$ is generic, ${ }^{\ddagger}$ but we have not been able to check this directly.

At the self-dual radius, it was shown in [14] that the most general solution to the factorisation constraint is parametrised by group elements in $\mathrm{SL}(2, \mathbb{C})$ rather than $\mathrm{SU}(2)$. In order to obtain the associated boundary states, one merely has to extend the representation matrix $D_{m, n}^{j}(g)$ in (3.2) accordingly. The computations from [14] can be carried over to show that these additional boundary conditions again satisfy Cardy's condition; however, the open string spectra in general involve complex conformal dimensions. Furthermore, in a free field construction, some of these branes would appear to be localised at imaginary positions. For these reasons, one is inclined to discard those additional boundary states as 'unphysical', at least for the purposes of string theory.

Apart from these unphysical branes the boundary states we have constructed above are the most general fundamental D-branes involving only degenerate Virasoro representations. The only other Virasoro Ishibashi states are in fact U(1) Ishibashi states. It therefore seems very plausible that the above branes, together with the usual Dirichlet and Neumann branes, from the complete set of fundamental conformal D-branes.

It is generally thought that all other boundary states are superpositions of such fundamental branes. In our case, however, we have to be more careful with the notion of superpositions than if we were to deal with a rational theory whose fundamental branes are given, say, by boundary states of the type constructed by Cardy in [5]. In that case, all further branes that are compatible with the Cardy states (i.e. give rise to positive integer linear combinations of characters in all the open string spectra) lie in (a positive cone of) the lattice spanned by these fundamental D-branes. This can be shown with the help of the simple (and purely algebraic) completeness criterion first formulated in [16]. In the present case, the corresponding statements are somewhat more subtle since we have to deal with a continuum of fundamental boundary conditions, and thus some analytic considerations come into play.

$\ddagger$ For non-generic group elements, i.e. if $a=0$ or $b=0$, the self-overlap contains several sectors with conformal dimension zero. Thus one does not expect the cluster condition to hold in the same form, and it is obvious from (4.13) that this will in fact not be the case. Compare also [11]. 
As mentioned before, our fundamental boundary states consist of the branes labelled by $g \in \mathrm{SU}(2) / \mathbb{Z}_{M} \otimes \mathbb{Z}_{N}$ with $a b \neq 0$, together with the conventional Dirichlet and Neumann $\mathrm{U}(1)$ boundary states (at arbitrary positions and Wilson lines). It is easy to check that this collection of boundary states is 'orthonormal' in the sense that the vacuum is only contained in the self-overlap of such a boundary state (where it occurs precisely once, meaning that they are fundamental), but not in the spectrum of open strings stretched between two different branes. Now let us consider an arbitrary conformal boundary state $\| B\rangle\rangle$ for the $c=1$ model at fractional radius $R$. Every such boundary state can be expanded in terms of the conformal Ishibashi states,

$$
\left.\left.\left.\| B\rangle\rangle=\sum_{p} B_{p}|p, p\rangle\right\rangle+\sum_{w} B_{w}|w,-w\rangle\right\rangle+\sum_{j, m, n} B_{(j ; m, n)}|j ; m, n\rangle\right\rangle
$$

where $B_{p}, B_{w}, B_{(j ; m, n)}$ are some finite constants and $\left.|p, p\rangle\right\rangle$ and $\left.|w,-w\rangle\right\rangle$ are the usual Dirichlet and Neumann Ishibashi states (with values of $p$ and $w$ appropriate for the radius $R$ ). We can then (at least formally) Fourier transform to write $\| B\rangle$ as

$$
\begin{gathered}
\left.\left.\left.\left.\| B\rangle\rangle=\int_{S_{\frac{1}{2 R}}^{1}} d \tilde{x}_{0} F_{N}\left(B ; \tilde{x}_{0}\right) \| N, \tilde{x}_{0}\right\rangle\right\rangle+\int_{S_{R}^{1}} d x F_{D}\left(B ; x_{0}\right) \| D, x_{0}\right\rangle\right\rangle \\
\left.\left.\quad+\int_{\mathrm{SU}(2)^{\prime}} d \mu(g) F_{\mathrm{SU}(2)}(B ; g) \| g\right\rangle\right\rangle
\end{gathered}
$$

where $\mathrm{SU}(2)^{\prime}=\mathrm{SU}(2) / \mathbb{Z}_{M} \times \mathbb{Z}_{N}$ (excluding the cases $a b=0$ ), and $d \mu(g)$ is the Haar measure. If $\| B\rangle\rangle$ is again one of the branes from above, the $F_{I}$ are delta-functions. More generally, the expansion (6.4) - along with the calculations from Section 4 leading to the open string partition function - make sense whenever the $F_{I}$ are tempered distributions. It therefore appears reasonable to take this as the condition that characterises the appropriate generalisation of the lattice of boundary states that arises for rational theories. In particular, the (unphysical) solutions to the factorisation condition, labelled by $\mathrm{SL}(2, \mathbb{C}) \backslash \mathrm{SU}(2)$, do not satisfy this criterion.

Until now we have only considered rational radii $R=\frac{M}{N} R_{\text {s.d. }}$, including the limiting cases $R=0$ and $R=\infty$. For 'non-rational' radii, the only Virasoro Ishibashi states that correspond to degenerate representations arise for $m=n=0$. The relevant matrix elements $D_{0,0}^{j}(g)$ then actually only depend on $a$ and not on $b$. If we introduce $x \in[0,1]$ by $x=2|a|^{2}-1$, we find

$$
D_{0,0}^{j}(g)=P_{j}(x),
$$


where $P_{j}(x)$ is the $j$ th Legendre polynomial. Indeed, it follows from (3.4) that the left hand side of $(6.5)$ is

$$
\begin{aligned}
D_{0,0}^{j}(g) & =\sum_{l=0}^{j}\left(\begin{array}{l}
j \\
l
\end{array}\right)\left(\begin{array}{c}
j \\
j-l
\end{array}\right)\left(|a|^{2}\right)^{j-l}\left(-|b|^{2}\right)^{l} \\
& =\frac{1}{2^{j}} \sum_{l=0}^{j}\left(\begin{array}{l}
j \\
l
\end{array}\right)\left(\begin{array}{c}
j \\
j-l
\end{array}\right)(x+1)^{j-l}(x-1)^{l} .
\end{aligned}
$$

On the other hand, the Rodriguez formula for Legendre polynomials (see for example [19]) implies that

$$
\begin{aligned}
P_{j}(x) & =\frac{1}{2^{j} j !}\left(\frac{d}{d x}\right)^{j}\left(x^{2}-1\right)^{j} \\
& =\frac{1}{2^{j} j !} \sum_{l=0}^{j}\left(\begin{array}{l}
j \\
l
\end{array}\right)\left(\frac{d}{d x}\right)^{l}(x+1)^{j}\left(\frac{d}{d x}\right)^{j-l}(x-1)^{j} \\
& =\frac{1}{2^{j}} \sum_{l=0}^{j}\left(\begin{array}{l}
j \\
l
\end{array}\right)(x+1)^{j-l}\left(\begin{array}{c}
j \\
j-l
\end{array}\right)(x-1)^{l},
\end{aligned}
$$

where we have used the Leibniz rule in the second line. The fact that the coefficients of the boundary states for an irrational radius are Legendre polynomials was already mentioned by Friedan [3]; an in-depth analysis of these boundary states (and in particular the determination of the relevant overlaps) is in preparation [20].*

\section{World-sheet supersymmetry}

We shall now exhibit boundary states similar to the $\| g\rangle\rangle_{M, N}$ from above for the analogous supersymmetric situation. We start from a bulk theory that consists of a compactified free boson and a free Majorana fermion, and use only Ishibashi states from degenerate representations of the $N=1$ super Virasoro algebra. The gluing conditions we impose on the boundary states $\| B, \eta\rangle\rangle$ are

$$
\left.\left.\left.\left(L_{n}-\bar{L}_{-n}\right) \| B, \eta\right\rangle=\left(G_{r}+i \eta \bar{G}_{-r}\right) \| B, \eta\right\rangle\right\rangle=0
$$

where $G_{r}$ are the modes of the supercurrent, and $\eta$ is a sign to be discussed later. In addition to checking Cardy's condition, we shall have to address questions related to the GSO projection. Moreover, at least when passing to consistent ten-dimensional superstring backgrounds, it will be interesting to see whether the new boundary states are stable.

^ We thank Romek Janik for sending us copies of his notes prior to publication. 
The partition function of the $c=\frac{3}{2}$ bulk theory made up from a free boson and a free Majorana fermion is a product

$$
Z_{\text {susy }}=Z_{\text {boson }} \cdot \frac{1}{2}\left\{\left|f_{3}(q)\right|^{2}+\left|f_{4}(q)\right|^{2}+\left|f_{2}(q)\right|^{2}\right\}
$$

of the partition function $Z_{\text {boson }}$ from $(2.2)$ and that of the two-dimensional Ising model; the $f_{i}$ are functions defined by

$$
\begin{aligned}
& f_{2}(q)=\sqrt{2} q^{\frac{1}{24}} \prod_{n=1}^{\infty}\left(1+q^{n}\right), \\
& f_{3}(q)=q^{-\frac{1}{48}} \prod_{n=1}^{\infty}\left(1+q^{n-\frac{1}{2}}\right), \\
& f_{4}(q)=q^{-\frac{1}{48}} \prod_{n=1}^{\infty}\left(1-q^{n-\frac{1}{2}}\right) .
\end{aligned}
$$

The first two terms in (7.2) describe the NS-NS sector (the sum of the two terms implements the GSO projection), while the last term is the contribution from the $\mathrm{R}-\mathrm{R}$ sector. For the above theory the GSO projection is $\frac{1}{2}\left(1+(-1)^{F+\bar{F}}\right)$, where $F$ and $\bar{F}$ are the leftand right-moving fermion number operators, rather than $\frac{1}{4}\left(1+(-1)^{F}\right)\left(1+(-1)^{\bar{F}}\right)$ as is familiar from the ten-dimensional superstring theories. The theory we are considering here is therefore the analogue of the type $0 \mathrm{~B}$ (or 0A) theory, rather than of the type IIB (or IIA) theory $[21,22]$. It may be worth mentioning that, for $c=\frac{3}{2}$, it is inconsistent to GSO project using the other GSO projection, because then the fermionic (twisted) sectors one has to introduce in order to obtain a modular invariant partition function do not obey level matching [23].

\subsection{The NS-NS sector}

Let us first concentrate on the NS-NS sector and collect some of the relevant facts concerning the representation theory of the $N=1$ superconformal algebra ( $S$ Vir) at $c=\frac{3}{2}$. In the NS sector the degenerate representations have conformal weight [24]

$$
h_{j}=\frac{j^{2}}{2}
$$

with $j=0,1,2, \ldots$. Such a representation $\mathcal{H}_{j}^{\text {SVir,NS }}$ has a null-vector at level $j+\frac{1}{2}$ that generates the whole subrepresentation, and its character is therefore given by

$$
\chi_{j}(q)=\left(\vartheta_{j}(q)-\vartheta_{j+1}(q)\right) f_{3}(q)
$$


The only sectors of the free boson and fermion theory that give rise to degenerate NSrepresentations of the $N=1$ superconformal algebra are those with $p_{L}=\left(\frac{\hat{m}}{2 R}+\hat{n} R\right)=$ $j \in \mathbb{Z}$, and analogously for $p_{R}$. Thus, only the radii

$$
R=\frac{M}{N}
$$

where $M$ and $N$ are coprime integers give rise to degenerate $S V$ ir representations. We also see that there is no radius where all $S$ Vir representations that occur in (7.2) are degenerate, the closest analogues of the self-dual radius from the bosonic case being $R=1$ and $R=\frac{1}{2}$, where half of the sectors yield degenerate representations.

For a radius as in eq. (7.6), the momenta take the form

$$
\left(p_{L}, p_{R}\right)=\left(\frac{\hat{m} N}{2 M}+\hat{n} \frac{M}{N}, \frac{\hat{m} N}{2 M}-\hat{n} \frac{M}{N}\right)=:(m, n) .
$$

The states with momentum and winding described by $(m, n) \in \mathbb{Z} \times \mathbb{Z}$ contain the representations $\mathcal{H}_{j}^{\text {SVir,NS }} \otimes \overline{\mathcal{H}}_{j}^{\text {SVir,NS }}$ provided that $j$ is an integer with $j \geq|m|,|n|$. Thus the possible Ishibashi states that involve these degenerate representations are labelled by the triplets $(j ; m, n)$ where $j$ is a non-negative integer, and $m$ and $n$ are integers satisfying the above inequality, and in addition by the sign $\eta= \pm 1$ from (7.1). These triplets correspond naturally to matrix elements of representations of $S O(3)$. As before we shall construct super Virasoro boundary states as linear combinations of the Ishibashi states $|j ; m, n ; \eta\rangle\rangle$, with coefficients given by $D_{m, n}^{j}(g)$.

As in the bosonic case, there are $M$ - and $N$-dependent conditions that tell us which $(m, n)$ actually occur, $c f$. (2.14). Evaluating the restriction $\left(p_{L}, p_{R}\right) \in \mathbb{Z} \times \mathbb{Z}$ carefully, one finds that only those degenerate $S$ Vir Ishibashi states are contained in the closed string theory for which the labels $m, n$ are integers satisfying

$$
\begin{aligned}
& m+n \equiv 0(\bmod N), \quad m-n \equiv 0(\bmod 2 M) \text { if } N \text { is odd, } \\
& m+n \equiv 0(\bmod N), \quad m-n \equiv 0(\bmod M) \quad \text { if } N \text { is even . }
\end{aligned}
$$

Given that both $m$ and $n$ are integers, $m+n$ is even if and only if $m-n$ is. Thus, distinguishing the cases is actually redundant in the NS-NS sector, but will become necessary in the R-R sector. In order to present all cases at once, we shall use the abbreviation

$$
\widetilde{M}:= \begin{cases}2 M & \text { if } N \text { is odd }, \\ M & \text { if } N \text { is even } .\end{cases}
$$

The restrictions (7.8) can be implemented with the help of the projectors (4.4) and (4.5) as before. Then the NS-NS parts of our $c=\frac{3}{2}$ boundary states can be written as

$$
\left.\| g, \eta\rangle\rangle_{\mathrm{NS}-\mathrm{NS}}=\sqrt{\frac{\widetilde{M} N}{2}} \sum_{j, m, n} D_{m, n}^{j}\left(P_{N}^{+} P_{\widetilde{M}}^{-}(g)\right)|j ; m, n ; \eta\rangle\right\rangle
$$


where $j, m, n$ are integers and $g \in \mathrm{SU}(2)$.

Before proceeding, we need to check whether the boundary state (7.10) is invariant under the GSO projection $\frac{1}{2}\left(1+(-1)^{F+\bar{F}}\right)$. It is clear from $(7.2)$ that the momentum and winding ground states are GSO invariant. In order to determine the fermion number of the Virasoro Ishibashi states, we consider the free boson and fermion representation with highest weight state labelled by $(m, n)$, which coincides with the $S$ Vir $\times S$ Vir Verma module with highest weight $(h, \bar{h})=\left(\frac{1}{2} m^{2}, \frac{1}{2} n^{2}\right)$. The first singular vector with respect to the left-moving $S$ Vir occurs at conformal weight $(h, \bar{h})=\left(\frac{1}{2}(m+1)^{2}, \frac{1}{2} n^{2}\right)$, and it is the highest weight state of an irreducible $S V$ ir $\times S V$ ir representation that occurs in the decomposition of the free boson and fermion representation in terms of $S V$ ir $\times S V i r$. Since the left-moving conformal weights of the two vectors differ by a half-odd integer, they have opposite left-moving fermion number. Using this argument repeatedly for left- and right-movers, we conclude that the Ishibashi state corresponding to $(j ; m, n)$ has eigenvalue $(-1)^{2 j-m-n}=(-1)^{m+n}$ under the GSO operator. Given (7.8), it then follows that (7.10) is indeed GSO invariant.

We have not specified the sign $\eta$ from our gluing conditions (7.1) yet. Indeed, the remarks in the previous paragraph show that we can choose $\eta$ freely in the NS-NS sector: if $\| g\rangle\rangle_{\mathrm{NS}-\mathrm{NS}}$ is GSO invariant, then

$$
\left.\left.\left.\left.(-1)^{F} \| g, \eta\right\rangle\right\rangle_{\mathrm{NS}-\mathrm{NS}}=(-1)^{\bar{F}} \| g, \eta\right\rangle\right\rangle_{\mathrm{NS}-\mathrm{NS}}
$$

because the two operators commute and are of order two. It follows that $\left.\left.(-1)^{F} \| g, \eta\right\rangle\right\rangle_{\mathrm{NS}-\mathrm{NS}}$ is another GSO invariant boundary state, which satisfies (7.1) with the opposite sign $-\eta$. As long as we focus on the NS-NS sector, we can therefore work with both sign choices.

Using the same techniques as before, we can compute the overlap between two such boundary states. For each $\alpha=\alpha_{k, l}$ that shows up, $c f$. (4.9), we have

$$
\begin{aligned}
\mathcal{A}^{\mathrm{NS}-\mathrm{NS}} & =\frac{1}{2} \sum_{j=0}^{\infty} \frac{\sin ((2 j+1) \alpha)}{\sin \alpha}\left(\vartheta_{j}(q)-\vartheta_{j+1}(q)\right) f_{3}(q) \\
& =\frac{1}{2} \vartheta_{0}(q) f_{3}(q)+\frac{1}{2} \sum_{j=1}^{\infty} \vartheta_{j}(q) f_{3}(q)\left[\frac{\sin ((2 j+1) \alpha)}{\sin \alpha}-\frac{\sin ((2 j-1) \alpha)}{\sin \alpha}\right] \\
& =\frac{1}{2} \sum_{j \in \mathbb{Z}} \cos (2 j \alpha) \vartheta_{j}(q) f_{3}(q)
\end{aligned}
$$


Upon a modular transformation, $f_{3}(-1 / \tau)=f_{3}(\tau)$, and therefore (7.12) becomes

$$
\begin{aligned}
\mathcal{A}^{\mathrm{NS}-\mathrm{NS}} & =\frac{1}{2} \int_{-\infty}^{\infty} d t \vartheta_{t}(\tilde{q}) f_{3}(\tilde{q}) \sum_{j \in \mathbb{Z}} e^{i(2 \pi t+2 \alpha) j} \\
& =\frac{1}{2} f_{3}(\tilde{q}) \int_{-\infty}^{\infty} d s \vartheta_{\frac{s}{2 \pi}}(\tilde{q}) \sum_{n \in \mathbb{Z}} \delta(s+2 \alpha+2 \pi n) \\
& =\frac{1}{2} \sum_{n \in \mathbb{Z}} \vartheta_{-\frac{\alpha}{\pi}+n}(\tilde{q}) f_{3}(\tilde{q}) .
\end{aligned}
$$

Up to the overall factor of $\frac{1}{2}$ (which will be necessary in order to implement the GSO projection in the open string sector), this defines a trace over a positive integer number of representations of the $N=1 \mathrm{NS}$ superconformal algebra.

\subsection{The $R-R$ sector}

Next we turn to the R-R sector. Here, the highest weight representations of the super Virasoro algebra can be characterised by the action of $G_{0}$ on the highest weight state, $G_{0}|\lambda\rangle=\lambda|\lambda\rangle$. The corresponding Verma module is degenerate provided that

$$
\lambda= \pm \lambda_{j}:= \pm \frac{j}{\sqrt{2}}
$$

for some $j \in \mathbb{N}_{0}+\frac{1}{2}$. The conformal weight of the ground state is then given by

$$
h_{j}^{R}=\frac{j^{2}}{2}+\frac{1}{16} .
$$

(Note that the 'Ramond ground states' of the theory - i.e. the states with $h=\frac{c}{24}-$ do not lie in degenerate representations of SVir.) The representation with $h_{j}$ as in (7.15) has a null-vector at level $j+\frac{1}{2}$, and the corresponding character is therefore proportional to

$$
\chi_{j}^{R}=f_{2}(q)\left(\vartheta_{j}(q)-\vartheta_{j+1}(q)\right) .
$$

Comparing (7.15) with the free boson spectrum (7.7) at radius $R=\frac{M}{N}$, one sees that a $\mathrm{U}(1) \times \mathrm{U}(1)$ sector labelled by half-odd-integer $m, n \in \mathbb{Z}+\frac{1}{2}$ gives rise to a degenerate $S$ Vir $\times S$ Vir representation $\mathcal{H}_{j}^{\mathrm{SVir}, \mathrm{R}} \otimes \overline{\mathcal{H}}_{j}^{\mathrm{SVir}, \mathrm{R}}$ for any $j \in \mathbb{Z}+\frac{1}{2}$ with $j \geq|m|,|n|$. The R-R part of the boundary states will therefore involve matrix elements $D_{m, n}^{j}$ of those $\mathrm{SU}(2)$ representations that do not define representations of $\mathrm{SO}(3)$.

Again, we shall use projectors $P_{N}^{+}$and $P_{\widetilde{M}}^{-}$to take care of the 'gaps' that arise from the restrictions ( $c f .(7.8))$

$$
m+n \equiv 0(\bmod N), \quad m-n \equiv 0(\bmod \widetilde{M}),
$$


where now $m, n \in \mathbb{Z}+\frac{1}{2}$. The R-R-part of our SU(2) family of SVir-preserving boundary states is thus given by

$$
\left.\| g, \eta\rangle\rangle_{\mathrm{R}-\mathrm{R}}=\sqrt{\frac{\widetilde{\widetilde{M} N}}{2}} \sum_{j, m, n} D_{m, n}^{j}\left(P_{N}^{+} P_{\widetilde{M}}^{-}(g)\right)|j ; m, n ; \eta\rangle\right\rangle .
$$

This looks formally like (7.10), but now $j, m, n$ take half-odd-integer values only.

Before proceeding we need to analyse the action of the fermionic zero modes (and the invariance under the GSO projection) carefully. Each momentum and winding number 'ground state' occurs with two-fold degeneracy since we have two fermionic zero modes $\psi_{0}$ and $\bar{\psi}_{0}$ that anti-commute. Let us introduce creation and annihilation operators $\psi^{ \pm}=$ $\frac{1}{\sqrt{2}}\left(\psi_{0} \pm i \bar{\psi}_{0}\right)$ which satisfy the standard anti-commutation relations

$$
\left\{\psi^{ \pm}, \psi^{ \pm}\right\}=0, \quad\left\{\psi^{ \pm}, \psi^{\mp}\right\}=1
$$

We can then choose a basis for the highest weight space to consist of states $\left|\left(p_{L}, p_{R}\right), \pm\right\rangle$ that are (up to normalisation) uniquely characterised by the conditions

$$
\psi^{\mp}\left|\left(p_{L}, p_{R}\right), \pm\right\rangle=0
$$

and we take the GSO operator to have eigenvalue \pm 1 on $\left|\left(p_{L}, p_{R}\right), \pm\right\rangle$, irrespective of the value of $\left(p_{L}, p_{R}\right)$. (This is the standard definition, compare for example [25].)

We are interested in boundary conditions that preserve the SVir symmetry algebra only, and thus we need to rewrite the relevant boundary condition in terms of $G_{0}$ and $\bar{G}_{0}$ rather than $\psi_{0}$ and $\bar{\psi}_{0}$. Let us therefore introduce the operators $G^{ \pm}=G_{0} \pm i \bar{G}_{0}$, which satisfy the anti-commutation relations

$$
\left\{G^{ \pm}, G^{ \pm}\right\}=0, \quad\left\{G^{ \pm}, G^{\mp}\right\}=2\left(L_{0}+\bar{L}_{0}\right)-\frac{1}{4}
$$

For the momenta and winding numbers we are considering above, the right hand side of the second equation is always non-zero (since $j=0$ is not allowed). Thus, for this set of representations, the space of ground states can also be thought of as being generated by $G^{ \pm}$. Depending on the choice of sign in the gluing conditions (7.1), the Ishibashi states we are interested in are annihilated by either $G^{+}$or $G^{-}$; in fact they satisfy $\left.G^{ \pm}|j ; m, n ; \pm\rangle\right\rangle=0$. In either case, the Ishibashi state is (up to normalisation) uniquely fixed by this condition since it fixes the ground state component.

On the ground states we have $G_{0}=\psi_{0} p_{L}$ and $\bar{G}_{0}=\bar{\psi}_{0} p_{R}$. Our conventions therefore imply that the state with GSO eigenvalue +1 is annihilated by $G^{-}$if $p_{L}=p_{R}$, but is 
annihilated by $G^{+}$if $p_{L}=-p_{R}$. In particular, this implies that the Ishibashi states $|j ; j, j ;-\rangle\rangle$ and $|j ; j,-j ;+\rangle\rangle$ are invariant under the GSO projection - and those with the opposite signs are not. Taking into account the relative fermion number of the various descendant SVir singular vectors (as before in the NS-NS case), we find that the Ishibashi state $|j ; m, n ; \pm\rangle$ has GSO eigenvalue $\mp(-1)^{m-n}$. Given (7.17) it then follows that for $N$ odd, all the relevant Ishibashi states $|j ; m, n ;-\rangle\rangle$ are GSO invariant, while for $N$ even, the same holds for the Ishibashi states $|j ; m, n ;+\rangle\rangle$. This demonstrates that our boundary states are GSO invariant as long as the suitable condition for the zero modes is chosen; the latter is dictated uniquely by the $\operatorname{sign} \eta$ in (7.1), so in contrast to the NS-NS sector there is only one family of GSO invariant R-R boundary states.

Overlaps between such R-R boundary states can be computed in an almost identical fashion as before: for each $\alpha=\alpha_{k, l}$ we get

$$
\begin{aligned}
\mathcal{A}^{\mathrm{R}-\mathrm{R}} & =\frac{1}{2} \sum_{j \in \mathbb{Z}+\frac{1}{2}} \cos (2 j \alpha) \vartheta_{j}(q) f_{2}(q) \\
& =\frac{1}{2} \int_{-\infty}^{\infty} \vartheta_{t}(\tilde{q}) f_{4}(\tilde{q}) \sum_{j \in \mathbb{Z}+\frac{1}{2}} e^{i(2 \pi t+2 \alpha) j}
\end{aligned}
$$

where we have normalised the Ishibashi states so that they give rise to $f_{2}(q)$ (rather than, say, $\left.\frac{1}{\sqrt{2}} f_{2}(q)\right)$. We can rewrite the last sum as

$$
\begin{aligned}
\sum_{j \in \mathbb{Z}+\frac{1}{2}} e^{i(2 \pi t+2 \alpha) j} & =e^{\frac{i}{2}(2 \pi t+2 \alpha)} 2 \pi \sum_{n \in \mathbb{Z}} \delta(2 \pi t+2 \alpha+2 \pi n) \\
& =2 \pi \sum_{n \in \mathbb{Z}}(-1)^{n} \delta(2 \pi t+2 \alpha+2 \pi n)
\end{aligned}
$$

Putting (7.23) back into (7.22) we then get

$$
\mathcal{A}^{\mathrm{R}-\mathrm{R}}=\frac{1}{2} \sum_{n \in \mathbb{Z}}(-1)^{n} \vartheta_{-\frac{\alpha}{\pi}+n}(\tilde{q}) f_{4}(\tilde{q}) .
$$

This corresponds to the trace over (one half of) the NS sector trace with the insertion of $\pm(-1)^{F}$.

Finally, the whole boundary state is a linear combination of the NS-NS and the R-R component,

$$
\left.\| g\rangle\rangle:=\| g, \eta\rangle\rangle_{\mathrm{NS}-\mathrm{NS}}+i \| g, \eta\right\rangle_{\mathrm{R}-\mathrm{R}},
$$

where the value of the parameter $\eta$ in the gluing condition for the two contributions is the same. In view of GSO invariance in the Ramond sector, the sign depends on the radius $R=\frac{M}{N}$ as $\eta=(-1)^{N}$. Taking the various results together, we obtain

$$
Z_{g_{1} g_{2}}(\tilde{q})=\left\langle\left\langle g_{1}\left\|q^{\frac{1}{2}\left(L_{0}+\bar{L}_{0}\right)-\frac{c}{12}}\right\| g_{2}\right\rangle\right\rangle=\sum_{k, l} \sum_{n \in \mathbb{Z}} \vartheta_{-\frac{\alpha_{k l}}{\pi}+n}(\tilde{q}) \frac{1}{2}\left(f_{3}(\tilde{q})-(-1)^{n} f_{4}(\tilde{q})\right)
$$


for the spectrum of open strings attached to the branes (7.25). This corresponds to a certain set of GSO projected NS-representations of SVir. As is familiar from the analysis of D-branes in type 0 , the open string only consists of bosonic degrees of freedom [25]. Not all different open string sectors in (7.26) have the same GSO projection, since the sign of the GSO projection depends on $n \bmod (2)$.

In addition to the boundary states (7.25) there exists another class of boundary states that are given by

$$
\| \hat{g}\rangle\rangle:=\sqrt{2} \| g, \eta\rangle\rangle_{\mathrm{NS}-\mathrm{NS}}
$$

where $\| g, \eta\rangle\rangle_{\mathrm{NS}-\mathrm{NS}}$ is defined by (7.10) and where now $\eta=-(-1)^{N}$. Here we have used the fact, explained around eq. (7.11), that the NS-NS boundary states are GSO invariant for both choices of $\eta$. The open string spectrum of (7.27) is

$$
Z_{\hat{g}_{1} \hat{g}_{2}}(\tilde{q})=\left\langle\left\langle\hat{g}_{1}\left\|q^{\frac{1}{2}\left(L_{0}+\bar{L}_{0}\right)-\frac{c}{12}}\right\| \hat{g}_{2}\right\rangle\right\rangle=\sum_{k, l} \sum_{n \in \mathbb{Z}} \vartheta_{-\frac{\alpha_{k l}}{\pi}+n}(\tilde{q}) f_{3}(\tilde{q})
$$

and therefore defines unprojected NS-representations of SVir. Thus the boundary states (7.27) are the analogues of Sen's non-BPS branes from type II [26] (see also [27]). The relative overlap between the two kinds of branes is also consistent since we find

$$
Z_{\hat{g}_{1} g_{2}}(\tilde{q})=\left\langle\left\langle\hat{g}_{1}\left\|q^{\frac{1}{2}\left(L_{0}+\bar{L}_{0}\right)-\frac{c}{12}}\right\| g_{2}\right\rangle\right\rangle=\sum_{k, l} \sum_{n \in \mathbb{Z}} \vartheta_{-\frac{\alpha_{k l}}{\pi}+n}(\tilde{q}) \frac{1}{\sqrt{2}} f_{2}(\tilde{q}) .
$$

Here we have used that the NS-NS overlap between two boundary states with different values of $\eta$ gives rise to the same formula as (7.12) except that $f_{3}(q)$ is replaced by $f_{4}(q)$; using the modular transformation of $f_{4}$, we then arrive at (7.29). Given the normalisation (7.3) of the function $f_{2}$, eq. (7.29) then describes an integer number of open string states (which define R-representations of SVir).

Similar to the bosonic case, the parameter space of the superconformal branes (7.25) is $\mathrm{SU}(2) / \mathbb{Z}_{\widetilde{M}} \times \mathbb{Z}_{N}$. For special values of $g \in \mathrm{SU}(2)$, the branes are superpositions of conventional Dirichlet or Neumann D-branes. For example, for odd $N$, the brane that corresponds to the identity matrix in $\mathrm{SU}(2)$ can be written in terms of $2 M=\widetilde{M}$ conventional Dirichlet branes that are equidistantly arranged around the circle and for which branes and antibranes alternate. ${ }^{\ddagger}$ On the other hand, the brane that corresponds to the matrix $\left(\begin{array}{ccc}0 & 1 \\ -1 & 0\end{array}\right)$ is described by $N$ (unstable) Neumann branes (whose Wilson lines take equidistant values) that are the analogues of Sen's non-BPS branes from type II [26] (see also [27]). Indeed,

$\ddagger$ For the case $M=1$, this configuration is similar to the non-BPS D-brane that was constructed in $[28]$. 
for this value of $g$, only Ishibashi states with $n=-m$ contribute to the boundary state, and if $N$ is odd, none of these satisfies (7.17); thus the boundary state has only a NS-NS component. (These non-BPS D-branes are however different from the non-BPS D-branes in (7.27) since the boundary states satisfy different gluing conditions corresponding to different values of $\eta$.)

For even $N$ the situation is reversed in that now the brane (7.25) that corresponds to the matrix $\left(\begin{array}{cc}0 & 1 \\ -1 & 0\end{array}\right)$ is described by $N$ conventional Neumann branes and anti-branes (whose Wilson lines take again equidistant values such that branes and anti-branes alternate), while the brane that is described by the identity matrix is described by $M$ unstable nonBPS Dirichlet branes (which are equidistantly localised around the circle). For two simple cases the relevant D-brane configurations are sketched in Figure 1.
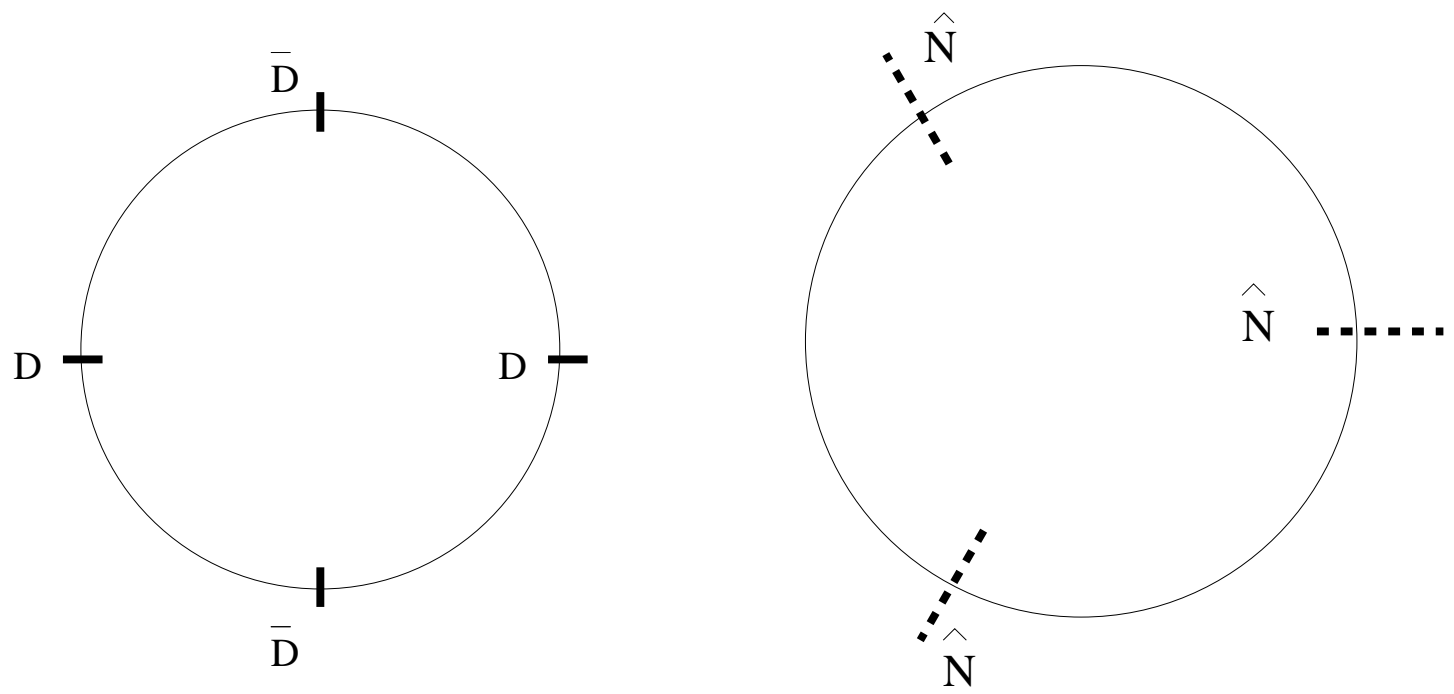

Fig. 0: The brane configurations for $R=2 / 3$ corresponding to special group elements: on the left, we have $g=$ id describing 2 Dirichlet branes $D$ and two antiDirichlet branes $\bar{D}$ distributed evenly over the target circle; on the right, $g=\left(\begin{array}{cc}0 & 1 \\ -1 & 0\end{array}\right)$, describing three non-BPS Neumann branes $\hat{N}$ with Wilson lines that are evenly distributed over the dual circle.

In either case, none of the branes carries non-trivial overall $\mathrm{R}-\mathrm{R}$ charge, since the zero-momentum R-R Ishibashi component (i.e. the $\mathrm{R}-\mathrm{R}$ Ishibashi state with $m=n=0$ ) is absent. Thus it is consistent for combinations of Dirichlet (or Neumann) branes to merge into non-BPS branes. Similar statements also hold for the other branch of boundary states that are described by (7.27); in this case, the space of boundary states is $\mathrm{SO}(3) / \mathbb{Z}_{\widetilde{M}} \times \mathbb{Z}_{N}$.

We have not attempted to check whether our superconformal boundary states satisfy the factorisation constraint briefly discussed in Section 6. The structural similarity with 
the bosonic case makes it tempting to believe that the supersymmetric generalisations are again consistent, and form a complete set (apart from possibly unphysical branes associated to $\mathrm{SL}(2, \mathbb{C}) \backslash \mathrm{SU}(2))$. However, the arguments from [14] used in Section 6 rely on the fact that the branch of the bulk moduli space that we were considering contains a rational point where Cardy's boundary states are available, along with an SU(2) family of marginal deformations. These are all held to be fully consistent and can be used to determine the structure constants of the classifying algebra, which do not depend on the circle radius. In contrast, the branch of the superconformal theories relevant for us does not have a point where the theory is rational with respect to an algebra containing $S V i r$. However, it may be possible to exploit the fact that this branch is connected to the superaffine line [23] where there is a point with $\widehat{\operatorname{su}}(2)_{2}$ symmetry. Similarly, one may be able to use this idea to make statements about conformal boundary states for $c=1$ orbifold theories.

Apart from this technical difficulty, there is also the more conceptual problem of what the relevant sewing relations for a GSO projected superconformal field theory are. Indeed, the relevant operator product in string theory must be GSO covariant, and therefore cannot simply be the naive conformal field theory operator product.

\section{Ten-dimensional boundary states, and stability}

We can use our boundary states from the $c=\frac{3}{2}$ theory to construct new boundary states for a full superstring background formed from ten copies of the free boson and free fermion system, roughly speaking by taking tensor products of the component boundary states. In contrast to ordinary Dirichlet branes, those boundary conditions break the $\mathrm{U}(1)^{10}$ symmetry, but clearly this procedure will still produce a rather special class of all possible superconformal boundary conditions for a ten-dimensional toroidal target. We shall nevertheless write down the 'tensor product' boundary states built from the states (7.25) from above since it is interesting to ask whether they are stable in the sense that no open string tachyons - i.e. boundary fields of total conformal dimension $h<\frac{1}{2}$ - occur in the spectrum. Moreover, the ten-dimensional setting offers a second possibility for the GSO projection (leading to type II string theory instead of type 0), and at the end of this section we shall discuss how to obtain GSO invariant boundary states for the type II theory.

For the time being, we apply the same GSO projection $\frac{1}{2}\left(1+(-1)^{F+\bar{F}}\right)$ as before, i.e. we work in a type 0 string theory (which is 'plagued' by a closed string tachyon, but is otherwise consistent). Also, let us use the light cone gauge and consider eight transverse directions only. When trying to build up eight-dimensional boundary states from those 
discussed in the previous section by taking tensor products, one has to bear in mind that modular invariance of the eight-dimensional bulk theory forbids any mixing of NS with R sectors from different $c=\frac{3}{2}$ component theories. Therefore, the tensor product has to be taken in NS-NS and R-R sectors separately, and the boundary states we consider have the form

$$
\left.\left.\left.\left.\left.\left.\left.\left.\| \vec{g}\rangle\rangle:=\| g_{1}\right\rangle\right\rangle_{\mathrm{NS}-\mathrm{NS}} \otimes \cdots \otimes \| g_{8}\right\rangle\right\rangle_{\mathrm{NS}-\mathrm{NS}}+i \| g_{1}\right\rangle\right\rangle_{\mathrm{R}-\mathrm{R}} \otimes \cdots \otimes \| g_{8}\right\rangle\right\rangle_{\mathrm{R}-\mathrm{R}}
$$

The self-overlap $Z_{\vec{g} \vec{g}}(\tilde{q})$ of such a boundary state can be calculated with the help of formulas (7.13) and (7.24) from the last section, and one finds

$$
Z_{\vec{g} \vec{g}}(\tilde{q})=\mathcal{A}_{\otimes}^{\mathrm{NS}-\mathrm{NS}}-\mathcal{A}_{\otimes}^{\mathrm{R}-\mathrm{R}}
$$

with

$$
\begin{aligned}
\mathcal{A}_{\otimes}^{\mathrm{NS}-\mathrm{NS}} & =\frac{1}{2^{8}} f_{3}^{8}(\tilde{q}) \prod_{i=1}^{8} \sum_{n_{i} \in \mathbb{Z}} \sum_{k_{i}, l_{i}} \vartheta_{-\frac{\alpha_{k_{i} l_{i}}}{\pi}+n_{i}}(\tilde{q}) \\
\mathcal{A}_{\otimes}^{\mathrm{R}-\mathrm{R}} & =\frac{1}{2^{8}} f_{4}^{8}(\tilde{q}) \prod_{i=1}^{8} \sum_{m_{i} \in \mathbb{Z}} \sum_{k_{i}, l_{i}}(-1)^{m_{i}} \vartheta_{-\frac{\alpha_{k_{i} l_{i}}}{\pi}+m_{i}}(\tilde{q}) .
\end{aligned}
$$

All indices $i$ run from 1 to 8 , and the range of $k_{i}$ and $l_{i}$ is determined by the radius $R_{i}$ as before. We have chosen the prefactor of the Ramond part of the boundary state in (8.1) so as to eliminate the vacuum state from the spectrum (8.3), but it is easy to see that, apart from the cases $a=0, M=1$ and $b=0, N=1$, the open string spectrum still contains states whose total conformal dimension is less than $\frac{1}{2}$. For example, the characters with $m_{i}=0$ for $i=1, \ldots, 7$ and $m_{8}= \pm 1$ occur with the 'wrong' GSO projection, and therefore (8.2) contains a state of conformal dimension $\frac{1}{2 \pi^{2}}\left(\alpha_{k_{8} l_{8}} \pm \pi\right)^{2}$. This corresponds to an open string tachyon for a suitable choice of $k_{8}$ and $l_{8}$ : for example, if $a b \neq 0$ we can choose $k_{8}=1$ and $l_{8}=0$ for $N_{8}>1$, or $l_{8}=1$ for $N_{8}=1$. In view of the fact that our boundary states are related to superpositions of brane anti-brane pairs, or to branes similar to Sen's non-BPS branes, it is not very surprising that they are unstable.

In the ten-dimensional case, we can also implement the more restrictive GSO projection $\frac{1}{4}\left[1+(-1)^{F}\right)\left(1 \pm(-1)^{\bar{F}}\right]$; the resulting theory is then type IIB/IIA string theory. In the following we shall restrict ourselves to discussing the situation for IIB; the analysis for IIA is completely analogous.

It turns out that only relatively minor modifications in the spectrum of branes occur when passing from type 0 to type IIB, since the definition of boundary states has to be adjusted only slightly in order to insure invariance under the more restrictive GSO 
projection. Suppose that some boundary state $\| B\rangle\rangle$, (for example $\| \vec{g}\rangle\rangle$ from before) is invariant under $(-1)^{F+\bar{F}}$. Since both $(-1)^{F}$ and $(-1)^{F}$ are of order two, it follows that

$$
\left.\left.\left.\left.(-1)^{F} \| B\right\rangle\right\rangle=(-1)^{\bar{F}} \| B\right\rangle\right\rangle
$$

Thus the combination

$$
\left.\left.\left.\left.\| B\rangle\rangle^{\mathrm{II}}=\frac{1}{\sqrt{2}}(\| B\rangle\right\rangle+(-1)^{F} \| B\right\rangle\right\rangle\right)
$$

is invariant under the more restrictive GSO projection of the type IIB theory. This boundary state $\| B\rangle\rangle^{I I}$ is non-trivial if $\left.\left.\| B\right\rangle\right\rangle$ is, because $\left.\left.\| B\right\rangle\right\rangle$ cannot be proportional to $\left.\left.(-1)^{F} \| B\right\rangle\right\rangle$; this follows from the fact that application of $(-1)^{F}$ to the boundary state switches the sign $\eta$ in the gluing condition (7.1) for the world-sheet fermions. On the other hand, different boundary states $\| B\rangle\rangle$ from type 0 will lead to the same $\| B\rangle{ }^{I I}$; examples are the states $\| B\rangle\rangle$ and $\left.\left.(-1)^{F} \| B\right\rangle\right\rangle$ from the type 0 theory. Thus we expect that in general there are more D-branes of type 0 than there are of type II, as is indeed the case for conventional D-branes [25].

The bosonic part of the open string spectrum of $\| B\rangle\rangle^{\mathrm{II}}$ is precisely the same as that of $\| B\rangle\rangle$ and, therefore, the stability analysis is as before. But open strings between $\| B\rangle{ }^{\mathrm{II}}$ branes will in addition have a Ramond sector. In order to see this, we first note that the overlap between $\left.\left.(-1)^{F} \| B_{1}\right\rangle\right\rangle$ and $\left.\left.(-1)^{F} \| B_{2}\right\rangle\right\rangle$ is the same as that between $\left.\left.\| B_{1}\right\rangle\right\rangle$ and $\left.\left.\| B_{2}\right\rangle\right\rangle$. Furthermore, the overlap between $\left.\left.\| B_{1}\right\rangle\right\rangle$ and $\left.\left.(-1)^{F} \| B_{2}\right\rangle\right\rangle$ vanishes in the R-R sector (since the ground states satisfy different gluing conditions for the zero modes), and in the NS-NS sector it leads to the expression from above except that now $f_{3}(q)$ is replaced by $f_{4}(q)$. Under a modular transformation, the functions $f_{4}(q)$ become $f_{2}(\tilde{q})$, and this gives rise to the R-sector of the open string.

\section{Conclusions and open problems}

In this paper we have constructed a family $\mathrm{SU}(2) / \mathbb{Z}_{M} \times \mathbb{Z}_{N}$ of conformal boundary states

for the theory of a free boson on a circle of rational radius $R=\frac{M}{N} R_{\text {s.d. }}$. These boundary states interpolate between combinations of Dirichlet and Neumann branes, but are fundamental, and symmetry breaking, for intermediate parameter values. We have shown that the relative overlaps between arbitrary elements of this family of boundary states satisfy Cardy's condition. We have also demonstrated that each of them satisfies the factorisation property at least for the subset of the bulk fields that correspond to degenerate Virasoro representations. Finally, we have proposed an analytic characterisation of the space of 
conformal boundary conditions that are spanned by these branes together with the usual Dirichlet and Neumann branes.

We have argued that the marginal operators that deform the branes along the moduli space are in fact truly marginal; it would be interesting to check this directly. It would also be interesting to verify that the branes we have constructed satisfy the other sewing constraints such as associativity of the boundary operator product expansion or, for that matter, the factorisation property for the non-degenerate bulk representations. One would then expect to be able to determine the most general (fundamental) boundary state along the lines of [14]. In fact, given the results of [14], it seems very plausible that the most general boundary conditions that satisfy the factorisation and Cardy constraints are described by the same formula we used here, but with $g$ being an arbitrary group element in $\mathrm{SL}(2, \mathbb{C})$ rather than in $\mathrm{SU}(2)$. The branes that are associated to $\mathrm{SL}(2, \mathbb{C}) \backslash \mathrm{SU}(2)$ do not satisfy the criterion discussed in Section 6 , as the $F_{I}$ appearing in an expansion in terms of our fundamental branes do not define distributions on the space of $C^{\infty}$-testfunctions. However, they also have imaginary couplings to the bulk fields, and are presumably not actually relevant in string theory. In this sense the branes that we have discussed in this paper are likely to form a complete spanning set for the physically relevant boundary states.

The boundary states that we have constructed depend critically on the precise value of the radius. (This simply reflects the fact that the same is true for the set of Virasoro Ishibashi states.) It would therefore be interesting to understand what happens to the boundary states as the radius is varied. One may guess that two of the marginal operators that are present for rational radii become relevant as the radius becomes irrational, and that the intermediate boundary states decay to combinations of conventional Dirichlet or Neumann boundary states, but the details remain to be worked out. The fact that these branes exist only for a discrete set of radii suggests that they do not have a straight-forward geometrical interpretation, but it would nevertheless be interesting to explore this issue.

We have also considered the theory of a free boson and a free fermion on a circle with $N=1$ world-sheet supersymmetry, and we have constructed a family of boundary states that preserve the corresponding super Virasoro algebra. While for the 'one-dimensional' case our boundary states are likely to form a complete set, the D-branes we have constructed for the ten-dimensional theory satisfy very special gluing conditions and are far from describing the most general case. (In fact, the D-branes we have constructed preserve the eight separate super Virasoro algebras associated to each of the eight directions in light-cone gauge, while in general only the diagonal super Virasoro subalgebra needs to be preserved.) One should try to investigate the general situation further. 
Another extension worth studying is that to $N=2$ world-sheet supersymmetry. All of the branes we have constructed are non-BPS and in fact unstable. This is not surprising since we have only preserved $N=1$ supersymmetry on the world-sheet, while one presumably needs to have $N=2$ supersymmetry on the world-sheet in order to obtain spacetime supersymmetric D-branes. It should therefore be worthwhile to analyse whether our construction can be generalised to the case where one preserves an $N=2$ super Virasoro algebra.

\section{Acknowledgements}

We are grateful to Gérard Watts for collaboration at an early stage of this work, and for many helpful comments and discussions. We also thank Romek Janik, Juan Maldacena, Daniel Roggenkamp, Katrin Wendland and Jean-Bernard Zuber for useful conversations. M.R.G. is grateful to the Royal Society for a University Research Fellowship, and the research of A.R. is supported in part by the Nuffield Foundation, Grant Number NUFNAL/00421/G. This work is also partly supported by EU contract HPRN-CT-2000-00122.

\section{References}

[1] J. Polchinski, Combinatorics of boundaries in string theory, Phys. Rev. D50, 6041 (1994); hep-th/9407031.

[2] J. Maldacena, G. Moore, N. Seiberg, Geometrical interpretation of D-branes in gauged WZW models, hep-th/0105038.

[3] D. Friedan, The space of conformal boundary conditions for the $c=1$ Gaussian model, unpublished note (1999).

[4] L. Birke, J. Fuchs, C. Schweigert, Symmetry breaking boundary conditions and WZW orbifolds, Adv. Theor. Math. Phys. 3, 671 (1999); hep-th/9905038.

[5] J.L. Cardy, Boundary conditions, fusion rules and the Verlinde formula, Nucl. Phys. B324, 581 (1989).

[6] J. Polchinski, L. Thorlacius, Free fermion representation of a boundary conformal field theory, Phys. Rev. D50, 622 (1994); hep-th/9404008.

[7] I.B. Frenkel, V.G. Kac, Basic representations of affine Lie algebras and dual resonance models, Invent. Math. 62, 23 (1981).

[8] G.B. Segal, Unitary representations of some infinite dimensional groups, Commun. Math. Phys. 80, 301 (1981).

[9] B.L. Feigin, D.B. Fuchs, Invariant skew-symmetric differential operators on the line and Verma modules over the Virasoro algebra, Funct. Anal. Appl. 16, 114 (1982); 
Verma modules over the Virasoro algebra, in: Lecture Notes in Mathematics 1060, Springer 1984.

[10] C.G. Callan, I.R. Klebanov, A.W. Ludwig, J.M. Maldacena, Exact solution of a boundary conformal field theory, Nucl. Phys. B422, 417 (1994); hep-th/9402113.

[11] A. Recknagel, V. Schomerus, Boundary deformation theory and moduli spaces of Dbranes, Nucl. Phys. B545, 233 (1999); hep-th/9811237.

[12] M.B. Green, M. Gutperle, Symmetry breaking at enhanced symmetry points, Nucl. Phys. B460, 77 (1996); hep-th/9509171.

[13] M. Hamermesh, Group theory and its applications to physical problems, AddisonWesley (1962).

[14] M.R. Gaberdiel, A. Recknagel, G.M.T. Watts, The conformal boundary states for $S U(2)$ at level 1 ; hep-th/0108102.

[15] D.C. Lewellen, Sewing constraints for conformal field theories on surfaces with boundaries, Nucl. Phys. B372, 654 (1992).

[16] G. Pradisi, A. Sagnotti, Y.S. Stanev, Completeness conditions for boundary operators in $2 d$ conformal field theory, Phys. Lett. B381, 97 (1996); hep-th/9603097.

[17] J. Fuchs, C. Schweigert, A classifying algebra for boundary conditions, Phys. Lett. B414, 251 (1997); hep-th/9708141.

[18] R.E. Behrend, P.A. Pearce, V.B. Petkova, J.-B. Zuber, Boundary conditions in rational conformal field theories, Nucl. Phys. B579, 707 (2000); hep-th/9908036.

[19] G. Arfken, Mathematical methods for physicists, Academic Press (1970).

[20] R.A. Janik, Exceptional boundary states at $c=1$, to appear.

[21] L.J. Dixon, J.A. Harvey, String theories in ten dimensions without spacetime supersymmetry, Nucl. Phys. B274, 93 (1986).

[22] N. Seiberg, E. Witten, Spin structures in string theory, Nucl. Phys. B276, 272 (1986).

[23] L. Dixon, P. Ginsparg, J. Harvey, $\hat{c}=1$ superconformal field theory, Nucl. Phys. B306, 470 (1988).

[24] J.D. Cohn, D. Friedan, Super characters and chiral asymmetry in superconformal field theory, Nucl. Phys. B296, 779 (1988).

[25] O. Bergman, M.R. Gaberdiel, A non-supersymmetric open string theory and S-duality, Nucl. Phys. B499, 183 (1997); hep-th/9701137.

[26] A. Sen, BPS D-branes on non-supersymmetric cycles, J. High Energy Phys. 9812, 021 (1998); hep-th/9812031.

[27] D.M. Thompson, Descent relations in type 0A/0B, hep-th/0105314.

[28] M. Gutperle, Non-BPS D-branes and enhanced symmetry in an asymmetric orbifold, J. High Energy Phys. 0008, 036 (2000); hep-th/0007126. 